\title{
The Applicability of the Ground Response Curve to Tunnelling Problems that Violate Rotational Symmetry
}

\author{
Journal Article \\ Author(s): \\ Schuerch, Roberto; Anagnostou, Georg \\ Publication date: \\ 2012-01 \\ Permanent link: \\ https://doi.org/10.3929/ethz-b-000043975
}

Rights / license:

In Copyright - Non-Commercial Use Permitted

Originally published in:

Rock Mechanics and Rock Engineering 45(1), https://doi.org/10.1007/s00603-011-0182-1 


\title{
The Applicability of the Ground Response Curve to Tunnelling Problems that Violate Rotational Symmetry
}

\author{
R. Schürch • G. Anagnostou
}

Received: 24 March 2011/Accepted: 30 August 2011/Published online: 27 September 2011

(C) Springer-Verlag 2011

\begin{abstract}
The applicability limits of the closed-form solution to the problem of ground response to tunnelling are sounded out by systematically investigating the effect of deviations from some of the important assumptions underlying the closed-form solution. The ground response curve (GRC) expresses the relationship between tunnel support pressure and the radial displacement of the tunnel boundary on the basis of a rotationally symmetric model. The assumptions underlying rotational symmetry are a circular tunnel, a hydrostatic and uniform initial stress field, an isotropic and homogeneous ground and uniformly distributed support pressure. Deviations from these assumptions generally necessitate potentially time-consuming numerical analyses. The paper revisits the classical problem of tunnel excavation in a linearly elastic, perfectly plastic ground obeying the Mohr-Coulomb yield criterion, and analyses the effects of non-uniformity and anisotropy of the initial stress field and of a non-circular tunnel geometry. The results show that the GRC also provides a reasonably accurate approximation of average tunnel convergence for a wide range of ground conditions that violate rotational symmetry.
\end{abstract}

Keywords Tunnelling - Ground response curve - Nonuniform initial stress field - Anisotropic initial stress field · Non-circular tunnel geometry

\section{List of symbols}

a Tunnel radius

$A_{0} \quad$ Cross-sectional area of the tunnel

C Depth of cover

R. Schürch $(\square) \cdot$ G. Anagnostou ETH Zurich, Zurich, Switzerland e-mail: roberto.schuerch@igt.baug.ethz.ch

$\begin{array}{ll}D & \text { Tunnel diameter } \\ E & \text { Young's modulus of the ground } \\ f_{\mathrm{c}} & \text { Uniaxial compressive strength of the ground } \\ H & \text { Horizontal convergence } \\ k & \text { Coefficient of lateral pressure } \\ k^{\prime} & \text { Normalized stress deviator } \\ l_{x}, l_{y l}, l_{y u} & \text { Dimensions of the computational model } \\ S & \text { Perimeter of the tunnel cross section } \\ s & \text { Tunnel boundary local co-ordinate } \\ u & \text { Displacement } \\ u_{\mathrm{A}}, u_{\mathrm{B}}, u_{\mathrm{C}} & \text { Radial displacements of characteristic points } \\ u_{\mathrm{FEM}} & \text { Average radial displacement of excavation } \\ & \text { boundary from FEM } \\ u_{\mathrm{GRC}} & \text { Radial displacement according to the GRC } \\ u_{n} & \text { Displacement component normal to the tunnel } \\ & \text { boundary } \\ u_{x, \text { axis }} & \text { Horizontal displacement at the tunnel axis } \\ u_{y, \text { crown }} & \text { Vertical displacement of the tunnel crown } \\ u_{y, \text { floor }} & \text { Vertical displacement of the tunnel floor } \\ V & \text { Vertical convergence } \\ x & \text { Co-ordinate } \\ y & \text { Co-ordinate }\end{array}$

Greek symbols

$\gamma \quad$ Unit weight of the ground

$\delta \quad$ Normalized difference between the horizontal

$\triangle A \quad$ Change of cross-sectional area

$\varepsilon_{u} \quad$ Error of the GRC

$v \quad$ Poisson's number

$\xi \quad$ Normalized local co-ordinate

$\sigma_{0} \quad$ Initial stress at the tunnel axis

$\sigma_{0}^{*} \quad$ Transformed initial stress

$\overline{\sigma_{0}} \quad$ Average initial stress at the tunnel axis

$\sigma_{\mathrm{H} 0} \quad$ Initial horizontal stress 


$\begin{array}{ll}\sigma_{\mathrm{V} 0} & \text { Initial vertical stress } \\ \sigma_{s} & \text { Support pressure } \\ \sigma_{s}^{*} & \text { Transformed support pressure } \\ \sigma_{y u} & \text { Vertical stress at the upper model boundary } \\ \varphi & \text { Angle of internal friction } \\ \psi & \text { Dilatancy angle }\end{array}$

\section{Introduction}

The ground response curve (GRC, Panet and Guellec 1974) is used to assess ground deformations and to analyse the interaction between ground and tunnel support. The existing closed-form solutions for the GRC are also useful for plausibility control with respect to the results of complex numerical calculations and for estimating the tunnel deformations occurring before support installation (Bernaud and Rousset 1996; Curran et al. 2003; Graziani et al. 2005; Oreste 2009; Svoboda and Mašín 2010).

The analytical solutions presented, e.g., by Panet and Guellec (1974) are based upon a rotationally symmetric model. The underlying assumptions are a circular and uniformly supported tunnel, a homogeneous and isotropic ground, and a hydrostatic and uniform initial stress field. As the condition of radial symmetry is rarely satisfied in tunnelling practice, however, researchers have also investigated ground response under conditions where one or more of the basic assumptions underlying radial symmetry are violated.

Detournay and Fairhurst (1987) investigated the influence of a non-hydrostatic stress field for a material obeying the Mohr-Coulomb failure criterion and determined the limit coefficient of lateral pressure for which the rotationally symmetric closed-form solution is reasonably accurate with respect to the extent of the failure zone and radial displacements. According to Detournay and Fairhurst (1987), the limit coefficient depends on the ratio of uniaxial compressive strength to initial stress. Carranza-Torres and Fairhurst (2000) followed a similar approach in their investigation into the ground reaction curve under an assumption of the Hoek-Brown failure criterion. The assumption of a hydrostatic initial stress field applies more to deep tunnels, where, as noticed by Eisenstein and Branco (1991), the variation with depth in the initial stresses is small in relation to the average stress for the elevation of the tunnel. The applicability of the GRC to shallow tunnels was studied by Vermeer et al. (2002) and Gonzáles-Nicieza et al. (2008). Vermeer et al. (2002) used an elasto-plastic constitutive model that involves softening, while Gonzáles-Nicieza et al. (2008) assumed a linearly elastic material behaviour and investigated the ground response for different tunnel shapes and a fixed ratio of horizontal to vertical stress of 0.8 . Based on the numerical results, they determined a series of corrective functions for estimating radial displacement.

The present paper revisits the question of GRC applicability by means of comparative numerical computations. More specifically, the paper systematically analyses the effects of tunnel geometry, anisotropy and non-uniformity in the initial stress field for a wide range of ground strength parameters, and shows that the analytical GRC represents a sufficiently accurate model for most practical purposes.

All numerical calculations have been carried out using the FEM-code HYDMEC developed at the ETH Zurich. The assumed constitutive behaviour is isotropic, linearly elastic and perfectly plastic according to the Mohr-Coulomb failure criterion with the non-associated plastic flow rule.

Sections 2 to 4 present separate analyses of each of the assumptions underlying radial symmetry: We start by investigating the effects of non-uniformity in the initial stress field and free surface (shallow tunnelling), while maintaining the assumptions of an isotropic initial stress tensor and a cylindrical tunnel (Sect. 2); we continue with the effects of initial stress anisotropy by considering a cylindrical tunnel in a uniform initial stress field (Sect. 3); and we close with an investigation into the influence of the tunnel's cross-sectional profile (circular, horseshoe or D-shaped) by assuming that the initial stress field is isotropic and hydrostatic (Sect. 4). Finally, Sect. 5 discusses a numerical example that violates all three of these assumptions simultaneously.

\section{Shallow Tunnel and Non-Uniform Initial Stress Field}

This section investigates the influence of the overburden $C$ on the convergences of an unsupported cylindrical tunnel (diameter $D$ ), assuming a hydrostatic stress field. The difference from the analytical GRC is due to the initial stress gradient (caused by the unit weight $\gamma$ of the ground) and the existence of a stress-free surface relatively close to the tunnel.

The stress gradient influences stress distribution in the vicinity of the opening. In shallow tunnels, the stress difference between the crown and the invert may be significant relative to the average stress prevailing at the tunnel axis. The boundary effect associated with the free surface is relevant particularly in the case of a low strength ground, because plastic stress redistribution around the opening practically ceases when the plastic zone reaches the ground surface. As discussed by Anagnostou (2001) and Vermeer et al. (2002), a minimum support pressure is then necessary to avoid collapse. At support pressures close to this critical value, the deformations grow asymptotically to infinity. In 
the model underlying the analytical GRC (an infinite disc without body forces), stress redistribution can occur without restraint, and an equilibrium is always possible (the only exception being that of an unsupported tunnel through cohesionless ground).

Figure 1 shows the model used for the numerical calculations. The dimensions of the model are $l_{x}=l_{y l}=$ $8 D$ and $l_{y u}=C$. To reduce computational effort, the vertical symmetry of the system is exploited. The upper model boundary represents the ground surface, which is considered to be stress-free $\left(\sigma_{y u}=0\right)$. The lateral far field boundary is fixed only in the horizontal direction. For the chosen size of the model, the effects of the far field boundaries are irrelevant. The initial vertical stress $\sigma_{\mathrm{V} 0}$ is equal to $\gamma\left(D / 2+l_{y u}-y\right)$, while the initial horizontal stress $\sigma_{\mathrm{H} 0}=\sigma_{\mathrm{V} 0}$ (i.e. the coefficient of lateral pressure $k=1)$. The computational domain was discretized by 3,600 isoparameric, 8-node elements with quadratic displacement shape functions. The finite element model has 14,701 nodes and 21,778 degrees of freedom.

As pointed-out by Poulos and Davis (1974), the closedform solution for a linearly elastic halfspace under line loading shows that "the displacements in a semi-infinite mass are only meaningful if evaluated as the displacement of one point relative to another point, neither point being located at infinity". This is also true for the linearly elastic, plane strain, shallow tunnel problem (Fig. 2c), because this is actually a generalization of the line or strip loading

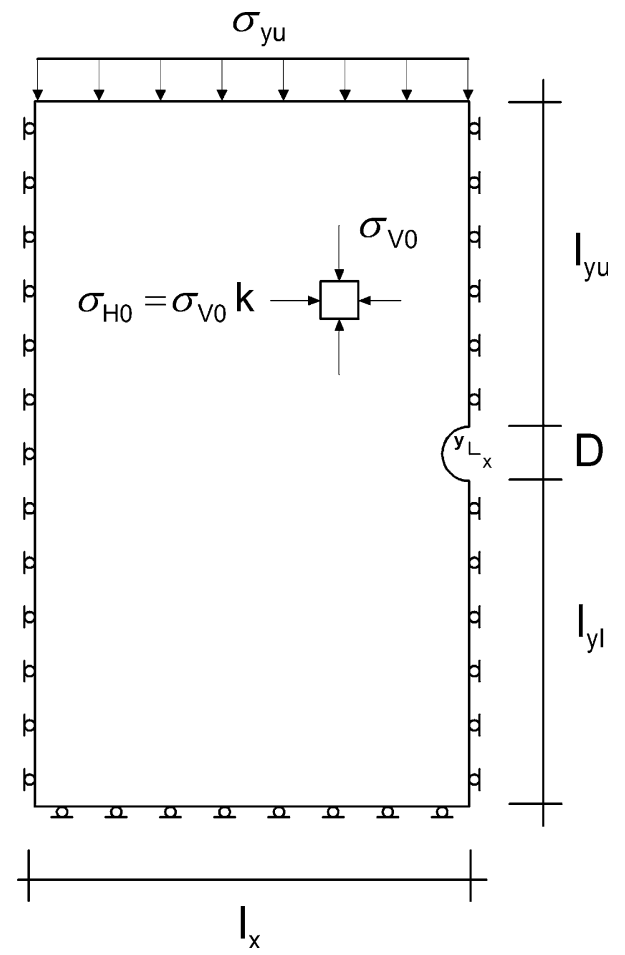

Fig. 1 Computational model problem (Fig. 2a, b, respectively). For this reason, we consider vertical convergence $V$ and horizontal convergence $H$ here rather than the absolute displacements:

$V=u_{y, \text { floor }}-u_{y, \text { crown }}, \quad H=2 u_{x, \text { axis }}$,

where $u_{y, \text { crown }}$ and $u_{y, \text { floor }}$ denote the vertical displacements (upwards positive) of the crown and of the floor, respectively, and $u_{x, \text { axis }}$ is the horizontal displacement of the tunnel wall (inwards positive).

To measure the error $\varepsilon_{u}$ introduced by the assumption of rotational symmetry, the deviation of the radial displacement $u_{\mathrm{GRC}}$ of the tunnel boundary (obtained from the closed form solution) from the average radial displacement $u_{\text {FEM }}$ (calculated by the FEM) is taken into account:

$\varepsilon_{u}=\frac{u_{\mathrm{GRC}}-u_{\mathrm{FEM}}}{u_{\mathrm{FEM}}}$,

where

$u_{\mathrm{FEM}}=0.25(H+V)$.

The deviation of the numerical results from rotational symmetry will be studied by considering the differences in convergence between the horizontal and vertical directions, expressed in terms of either the ratio $\mathrm{V} / \mathrm{H}$ or the normalized difference $\delta$ :

$\delta=\frac{V-H}{0.5(V+H)}$.

All displacement components, including those appearing in Eqs. 1 to 3, depend on the geometric parameters of the problem $(C, D)$ and the material constants of the ground (i.e. the unit weight $\gamma$, the Young's modulus $E$, the Poisson's ratio $v$, the uniaxial strength $f_{\mathrm{c}}$, the friction angle $\varphi$ and the dilatancy angle $\psi)$. In general,

$u=f\left(C, D, \gamma, E, v, f_{\mathrm{c}}, \varphi, \psi\right)$,

where $u$ denotes the displacement of an arbitrary point in an arbitrary direction. For dimensional reasons, and due to a general property of elasto-plastic continua, according to which the displacements depend linearly on $1 / E$ (Anagnostou and Kovári 1993), the general Eq. 5 can also be written in the following way:

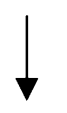

(a)

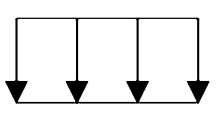

(b)

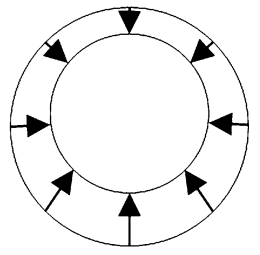

(c)
Fig. 2 Elastic halfspace a under line loading, b under strip loading, and $\mathbf{c}$ with excavation induced unloading of the tunnel boundary 
$\frac{E u}{\sigma_{0} D}=f\left(\frac{f_{\mathrm{c}}}{\sigma_{0}}, \frac{C}{D}, v, \varphi, \psi\right)$,

where $\sigma_{0}$ denotes the initial stress at the depth of the tunnel axis, i.e. $\sigma_{0}=(C+D / 2) \gamma$. On account of Eqs. 2, 4 and 6, the error $\varepsilon_{u}$ and the convergence difference $\delta$ depend only on $f_{\mathrm{c}} / \sigma_{0}, C / D, v, \varphi$ and $\psi$.

Figure $3 \mathrm{a}$ and $\mathrm{b}$ shows the error $\varepsilon_{u}$ and the convergence difference $\delta$, respectively, as a function of the ratio $C / D$ and the normalized uniaxial ground strength $f_{\mathrm{c}} / \sigma_{0}$. Figure 4 provides a more complete picture of the behaviour of the model by presenting the deformed tunnel shape and the extent of the overstressed region in four cases (indicated by the points A, B, C and D in the diagrams of Fig. 3). Cases $\mathrm{A}, \mathrm{B}$ and $\mathrm{C}$ illustrate the effect of the uniaxial compressive strength of the ground (for a fixed depth of cover), while cases C and D show the effect of depth of cover (for the same normalized strength $f_{\mathrm{c}} / \sigma_{0}$ ).

According to Fig. 3a, the smaller the depth of cover and the lower the strength of the ground, the larger will be the error introduced by the assumption of radial symmetry. Due to the asymmetric failure mechanism (Fig. 4, case C),

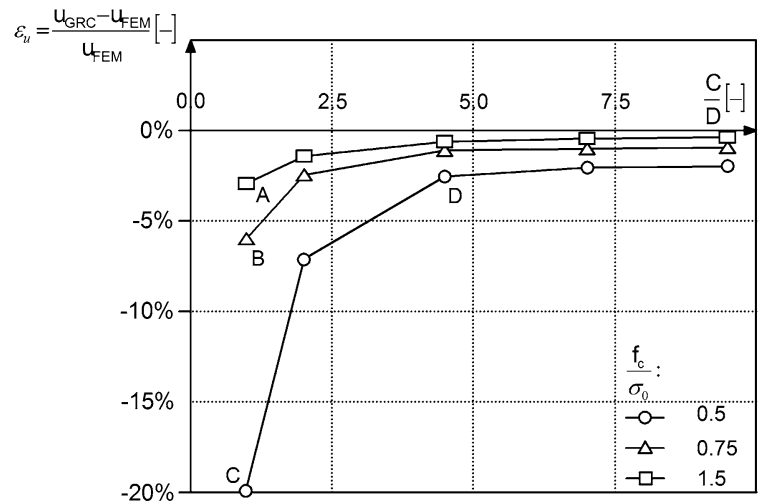

(a)

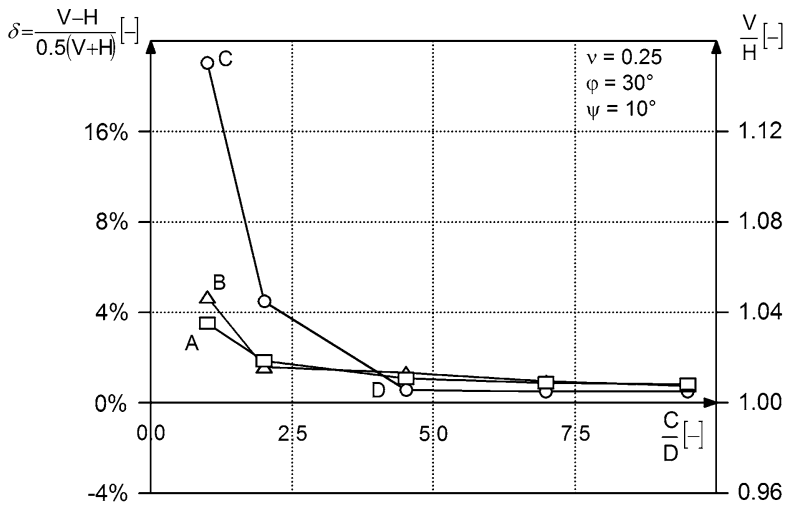

(b)

Fig. 3 Shallow unsupported cylindrical tunnel: a error $\varepsilon_{u}$ of the GRC and $\mathbf{b}$ normalized difference $\delta$ of vertical and horizontal convergence as a function of the normalized overburden $C / D$ and of the normalized ground strength $f_{\mathrm{c}} / \sigma_{0}$ the deviation between vertical and horizontal convergence also increases with decreasing overburden and ground strength. In case $\mathrm{C}$, where $\mathrm{C} / D=1$ and $f_{\mathrm{c}} / \sigma_{0}=0.5$, the error amounts to $20 \%$ (Fig. 2a). Figure 4 shows that in this case the plastic zone reaches the ground surface. It should be noted that the calculation failed to reach equilibrium in case $\mathrm{C}$ and, consequently, the actual error $\varepsilon_{u}$ is even bigger than indicated by Fig. 3a.

As long as the ground strength and the overburden are not too low, the effect of the free surface is not great and the deviation of the closed-form solution from the numerical results is small, despite the underlying assumption of rotational symmetry. Figure 3 indicates that at $C / D$-ratios higher than about 4 (i.e. at depths of 40-50 m for typical cross sections of traffic tunnels) a tunnel can be considered deep in the sense that the initial stress gradient and the free surface do not play an important role anymore.

\section{Non-Hydrostatic Initial Stress Field}

A cylindrical unlined deep tunnel is considered in a uniform but non-hydrostatic initial stress field. Based upon Anagnostou and Kovári (1993) as before, the following general relationship connects the displacements with the parameters of the problem:

$\frac{E u}{\overline{\sigma_{0}} D}=f\left(\frac{f_{\mathrm{c}}}{\overline{\sigma_{0}}}, k, v, \varphi, \psi\right)$,

where $\overline{\sigma_{0}}$ denotes the average initial stress at the tunnel axis:

$\overline{\sigma_{0}}=\frac{\sigma_{\mathrm{V} 0}+\sigma_{\mathrm{H} 0}}{2}=\sigma_{\mathrm{V} 0} \frac{(1+k)}{2}$.

Due to the symmetry of the problem, the results obtained for a specific value $k$ of the lateral stress coefficient can also be applied to the case with the coefficient $1 / k$ by rotating the axes by $90^{\circ}$ (Carranza-Torres and Fairhurst 2000). Instead of $k$, the normalized stress deviator $k^{\prime}$ may also be considered as a measure of the anisotropy of the initial stress tensor:

$k^{\prime}=\frac{\left|\sigma_{\mathrm{V} 0}-\sigma_{\mathrm{H} 0}\right|}{0.5\left(\sigma_{\mathrm{V} 0}+\sigma_{\mathrm{H} 0}\right)}=2 \frac{|1-k|}{1+k}$.

The computational model of Fig. 1 is considered again, but with the following differences from the last Section: all far field boundaries are located at a distance of $l_{x}=l_{y l}=l_{y u}=8 D$; the model is initialized with a uniform stress $\left(\sigma_{\mathrm{V} 0}, \sigma_{\mathrm{H} 0}\right)$; the initial vertical stress is prescribed as a boundary condition at the upper boundary $\left(\sigma_{y u}=\sigma_{\mathrm{V} 0}\right)$. The numerical calculations were carried out for normalized stress deviators $k^{\prime}$ between 0 and 0.66 (i.e., $0.5<k<2$ ), for normalized compressive strengths 


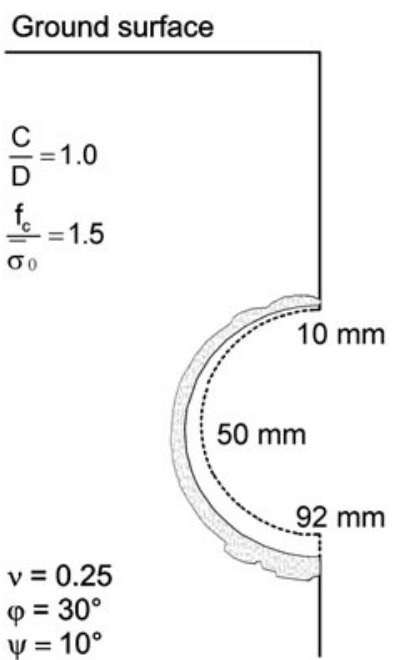

(a)

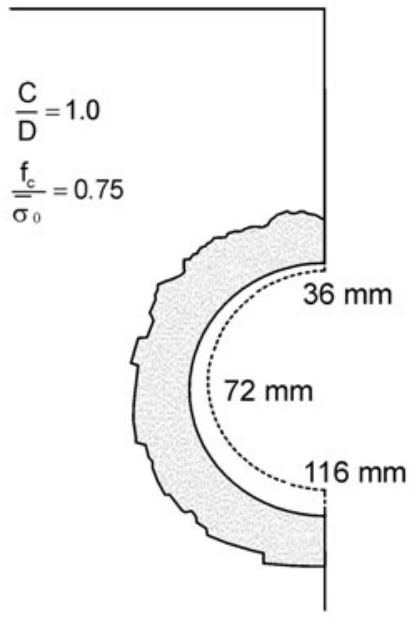

(b)

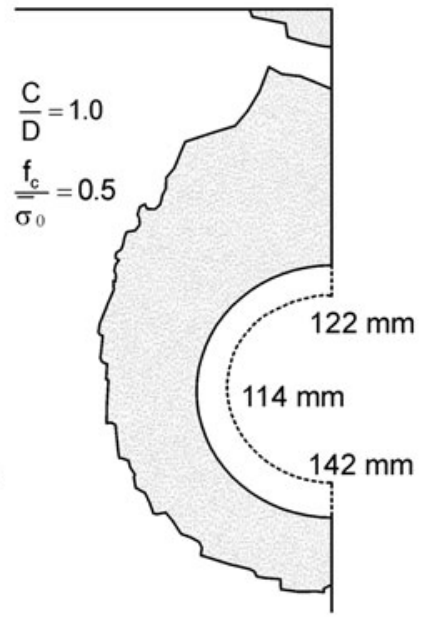

(c)

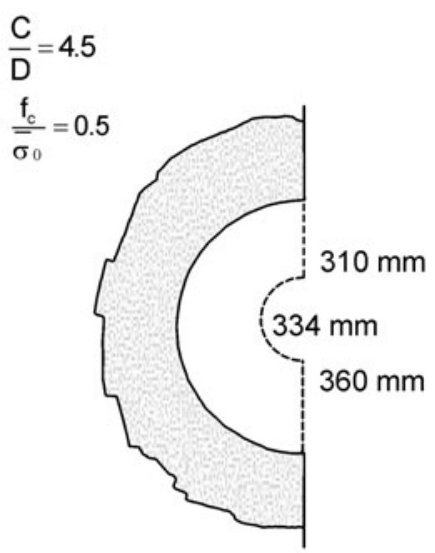

(d)

Fig. 4 Shallow unsupported cylindrical tunnel: deformed tunnel shape and extent of the overstressed region for four cases indicated by the points A B, C and D in Fig. 3 (parameters like Fig. 3, displacement values for $\left.D=10 \mathrm{~m}, E=100 \mathrm{MPa}, \overline{\sigma_{0}}=\gamma \mathrm{D}(C / D+0.5), \gamma=25 \mathrm{kN} / \mathrm{m}^{3}\right)$

$f_{\mathrm{c}} / \overline{\sigma_{0}}=0.2-3$ and for the friction angles $\varphi$ of $20^{\circ}, 30^{\circ}$ and $40^{\circ}$. Figure 5 presents the numerical results in the same manner as in Sect. 2, the only difference being that the abscissae contains the normalized stress deviator $k^{\prime}$ (or, which is equivalent, the coefficient of the lateral stress $k$ ) as independent parameter. The effect of the friction angle $\varphi$ (all other parameters remaining constant) is rather small even in the case of overstressed ground $\left(f_{\mathrm{c}} / \overline{\sigma_{0}}=0.2\right)$ and it disappears, of course, if the behaviour is elastic or almost elastic $\left(f_{\mathrm{c}} / \overline{\sigma_{0}}>0.5\right)$.

Let us consider first the special case of linearly elastic behaviour. In this case the principle of superposition is valid, i.e. the boundary displacements are equal to the sum of the displacements caused by the hydrostatic part of the initial stress and those caused by the purely deviatoric part of the initial stress. Assuming (for simplicity and without loss of generality) that $k<1$, the decomposition of the initial stress into these two parts reads as follows:

$$
\begin{aligned}
\left\{\begin{array}{l}
\sigma_{\mathrm{H} 0} \\
\sigma_{\mathrm{V} 0}
\end{array}\right\} & =\left\{\begin{array}{l}
\frac{\sigma_{\mathrm{V} 0}+\sigma_{\mathrm{H} 0}}{2} \\
\frac{\sigma_{\mathrm{V} 0}+\sigma_{\mathrm{H} 0}}{2}
\end{array}\right\}+\left\{\begin{array}{c}
-\frac{\sigma_{\mathrm{V} 0}-\sigma_{\mathrm{H} 0}}{2} \\
\frac{\sigma_{\mathrm{V} 0}-\sigma_{\mathrm{H} 0}}{2}
\end{array}\right\} \\
& =\overline{\sigma_{0}}\left(\left\{\begin{array}{l}
1 \\
1
\end{array}\right\}+\left\{\begin{array}{c}
-\frac{k \prime}{2} \\
\frac{k \prime}{2}
\end{array}\right\}\right) .
\end{aligned}
$$

The hydrostatic part produces a uniform convergence, which is proportional to the average initial stress $\overline{\sigma_{0}}$. The deviatoric part produces only antisymmetric deformations, i.e. the horizontal and vertical convergences have the same magnitude, but opposite signs, and are proportional to the normalized initial stress deviator $k^{\prime}$ (Eq. 10). This is why in the case of a high normalized strength the normalized difference $\delta$ between the vertical and horizontal convergences increases linearly with $k^{\prime}$ (see the lines for $f_{\mathrm{c}} / \overline{\sigma_{0}}=3$ in Fig. $5 \mathrm{~d}-\mathrm{f}$ ), while the average displacement error $\varepsilon_{u}$ of the GRC is equal to zero, independently of the initial stress deviator $k^{\prime}$ (see lines for $f_{\mathrm{c}} / \overline{\sigma_{0}}=3$ in Fig. 5a-c).

In general, the error $\varepsilon_{u}$ of the GRC increases with the initial stress deviator $k^{\prime}$ and with the degree of overstressing, i.e., with decreasing values of normalized strength $f_{\mathrm{c}} / \overline{\sigma_{0}}$ (Fig. 5a-d). The effect of strength on the error $\varepsilon_{u}$ is nevertheless small at $f_{\mathrm{c}} / \overline{\sigma_{0}}$-ratios lower than 1 . In the case of overstressed ground $\left(0.2<f_{\mathrm{c}} / \overline{\sigma_{0}}<1\right)$ and high $k^{\prime}$ values, the error $\varepsilon_{u}$ reaches $20-30 \%$ (the maximum value applies to $k^{\prime}=2 / 3$, which corresponds either to a very low or to a very high lateral pressure coefficient $k$ of 0.5 or 2 , respectively). For the range $k=0.7-1.5$ suggested by Carranza-Torres and Fairhurst (2000), Fig. 5 shows that the maximum error $\varepsilon_{u}$ amounts to just $15 \%$. These results agree with Detournay and Fairhurst (1987), according to which the closed-form, rotationally symmetric solution approximates reasonably well to the extent of the overstressed region and the magnitude of the average convergence if $k>0.6$ (or, equivalently, if $k^{\prime}<0.4$ ). The validity of these results was also confirmed by Carranza-Torres and Fairhurst (2000) for the case of a material obeying the HoekBrown failure criterion.

As expected, the difference $\delta$ between the horizontal and vertical convergence increases with the initial stress deviator $k^{\prime}$, but its sign depends on the normalized strength $f_{\mathrm{c}} / \overline{\sigma_{0}}$, i.e. on the degree of overstressing. The reasons for this are well understood since Detournay (1983): In the case of an elastic or almost elastic response, the greatest convergence occurs in the direction of the maximum unloading, i.e. in the direction of the highest principal initial stress (this is the horizontal direction in the example of Fig. 6a, where 


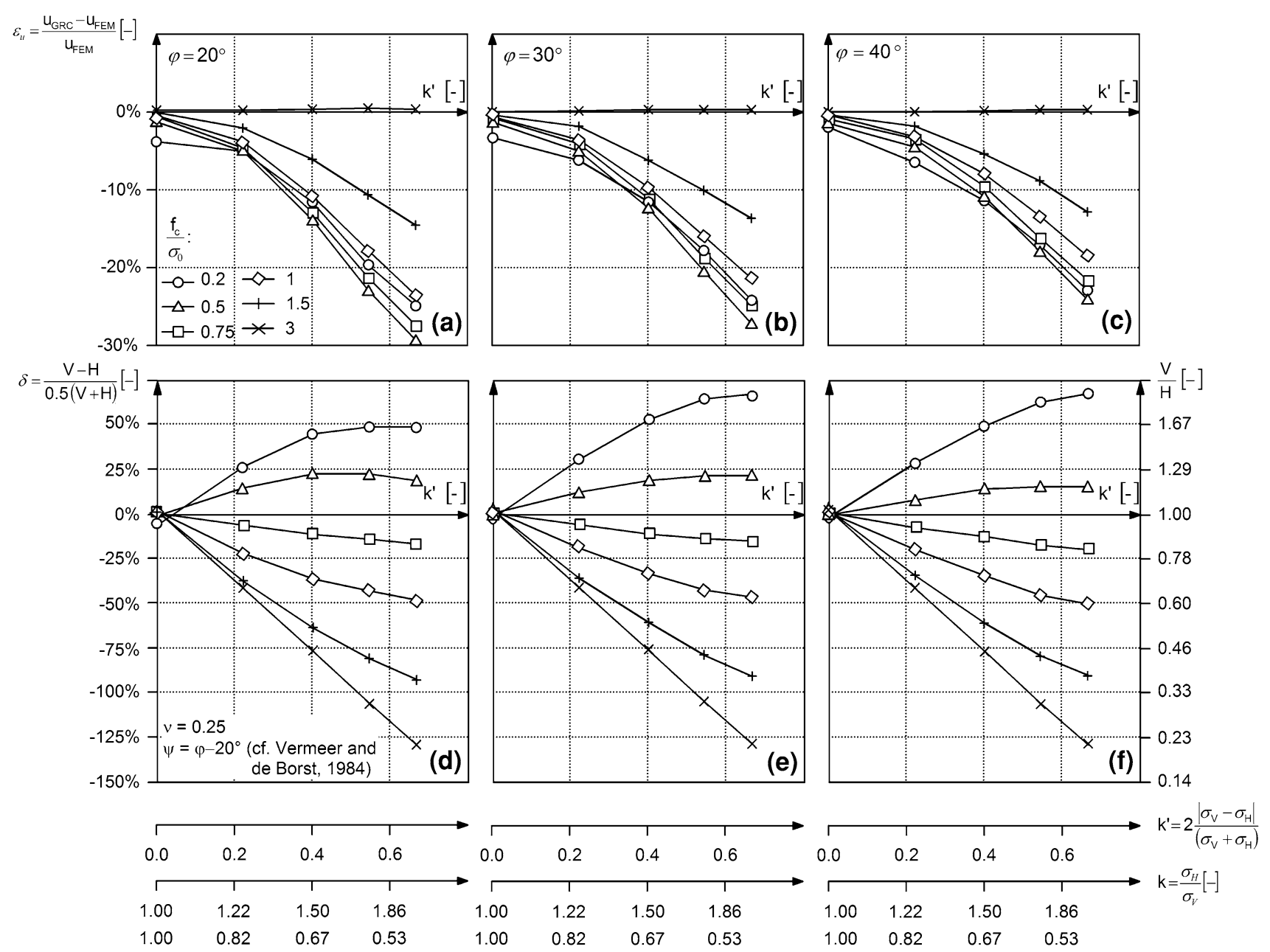

Fig. 5 Deep cylindrical tunnel in non-hydrostatic initial stress field: a-c Error $\varepsilon_{u}$ of the GRC and $\mathbf{d}-\mathbf{f}$, normalized difference $\delta$ of vertical and horizontal convergence for $\varphi=20^{\circ}, 30^{\circ}$ and $40^{\circ}$ as a function of

$k=2$ ). This changes in the case of low normalized strength $f_{c} / \overline{\sigma_{0}}$, because the ground becomes more overstressed and experiences larger plastic deformations in the zones of high initial tangential stress (above the crown and beneath the floor in the example of Fig. 6b), thus squeezing more in the direction of the lowest initial stress (i.e. in the vertical direction in the example of Fig. 6b).

The greatest convergence differences $\delta$ between the horizontal and vertical directions occur either in the case of a practically elastic response (which is not problematic from an engineering point of view) or in the case of heavily overstressed ground (which is rather rare). Apart from these cases, the normalized difference between the vertical and horizontal displacement is less than $50 \%$.

\section{Non-Circular Tunnel Cross Section}

Next we investigate the influence of the tunnel shape, while maintaining the homogeneity and isotropy of the initial the normalized initial stress deviator $k^{\prime}$ and of the normalized compressive strength of the ground $f_{c} / \overline{\sigma_{0}}$

stress field. More specifically, we compare the convergences of a horseshoe-shaped or D-shaped profile (Fig. 7b, c, respectively) to those of the reference case (a circular cross section, Fig. 7a), for which the GRC is valid in a strict mathematical sense. Figure 7 shows the deformed tunnel cross section, the plastic zone and the distribution of the plastic strain along the symmetry axis beneath the invert for a given parameter set (material constants, tunnel size and initial stress) and for the three shapes. The very small plastic strains developing in the zone immediately beneath the flat invert (Fig. 7b, c) are due to out-of-plane plastic flow.

Due to the non-uniform displacements in a non-circular excavation boundary, we treat the change in the crosssectional area $\Delta A$ of the tunnel as an overall measure of convergence. Neglecting second order terms,

$\Delta A=\int_{S} u_{n} \mathrm{~d} s$, 


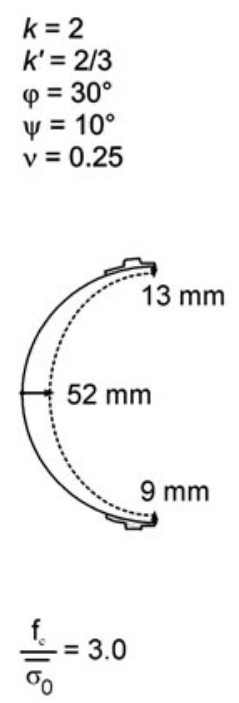

(a)

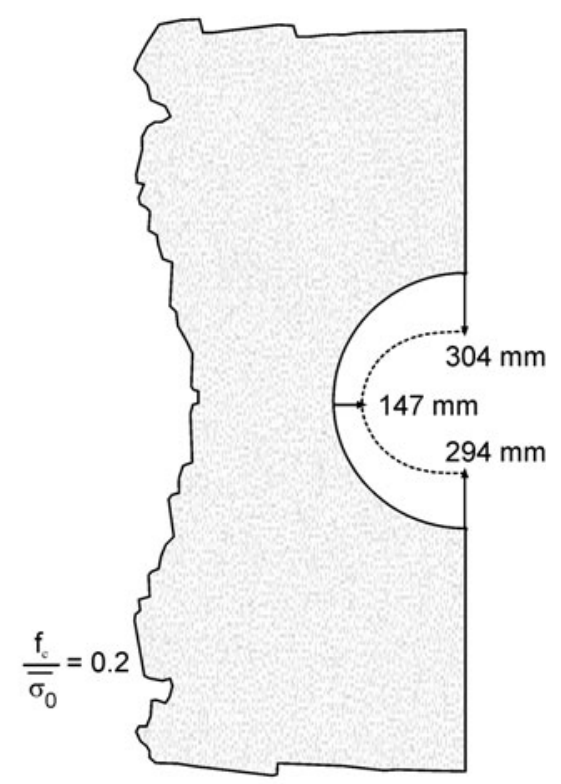

(b)

Fig. 6 Deep unsupported cylindrical tunnel in non-hydrostatic initial stress field: deformed tunnel cross section and extent of the plastic zone for a high and for a low strength of the ground (displacement values for $D=10 \mathrm{~m}, E=1 \mathrm{GPa}, \overline{\sigma_{0}}=2.5 \mathrm{MPa}$ )

where $S, s$ and $u_{n}$ denote the perimeter of the tunnel cross section, the local co-ordinate and the displacement component normal to the tunnel boundary, respectively (Fig. 7c).

Based upon Anagnostou and Kovári (1993),

$\frac{E u_{n}}{\sigma_{0}^{*} a}=f_{1}\left(\frac{\sigma_{s}^{*}}{\sigma_{0}^{*}}, v, \varphi, \psi, \frac{s}{a}\right)$,

where $a$ is a characteristic length (here taken equal to the radius of a circle having the same cross sectional area as the tunnel cross section under consideration), while $\sigma_{s}^{*}$ and $\sigma_{0}^{*}$ are Caquot's (1934) transformations of the support pressure $\sigma_{s}$ and of the initial stress $\sigma_{0}$, respectively:

$$
\begin{gathered}
\sigma_{s}^{*}=\sigma_{s}+\frac{c}{\tan \varphi}=\sigma_{s}+\frac{1-\sin \varphi}{2 \sin \varphi} f_{\mathrm{c}}, \\
\sigma_{0}^{*}=\sigma_{0}+\frac{c}{\tan \varphi}=\sigma_{0}+\frac{1-\sin \varphi}{2 \sin \varphi} f_{\mathrm{c}}
\end{gathered}
$$

From Eqs. (11) and (12) we obtain the following dimensionless expression:

$\frac{E}{\sigma_{0}^{*}} \frac{\Delta A}{A_{0}}=\frac{1}{\pi} \int_{S / a} f_{1}\left(\frac{\sigma_{s}^{*}}{\sigma_{0}^{*}}, v, \varphi, \psi, \xi\right) d \xi=f_{2}\left(\frac{\sigma_{s}^{*}}{\sigma_{0}^{*}}, v, \varphi, \psi\right)$,

where $A_{0}$ denotes the cross sectional area $\left(A_{0}=\pi a^{2}\right)$ and $\xi=s / a$. For fixed values of the material constants $v, \varphi$ and $\psi$, Eq. (14) represents a generalized GRC because it connects the overall convergence $(E \Delta A) /\left(\sigma_{0}^{*} A_{0}\right)$ with the normalized support pressure $\sigma_{s}^{*} / \sigma_{0}^{*}$. In the special case of a circular cross section, the displacement distribution is uniform $\left(u_{n}\right.$ is equal to the radial displacement $u_{\mathrm{GRC}}$ and does not depend on the coordinate $s): S=2 \pi a$ and $\Delta A / A_{0}=2 u_{\mathrm{GRC}} / a$.

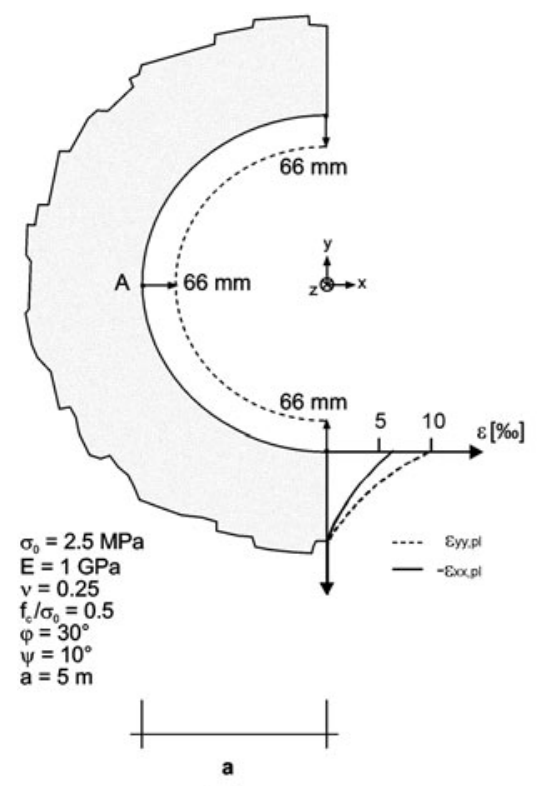

(a)

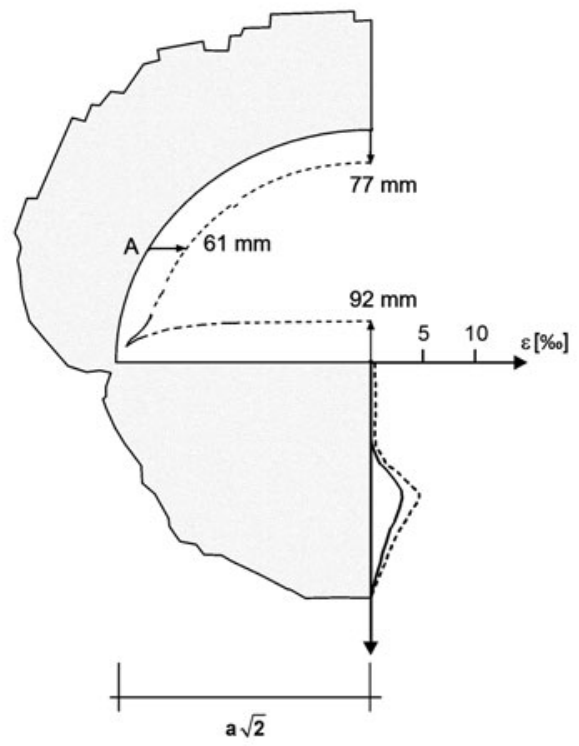

(b)

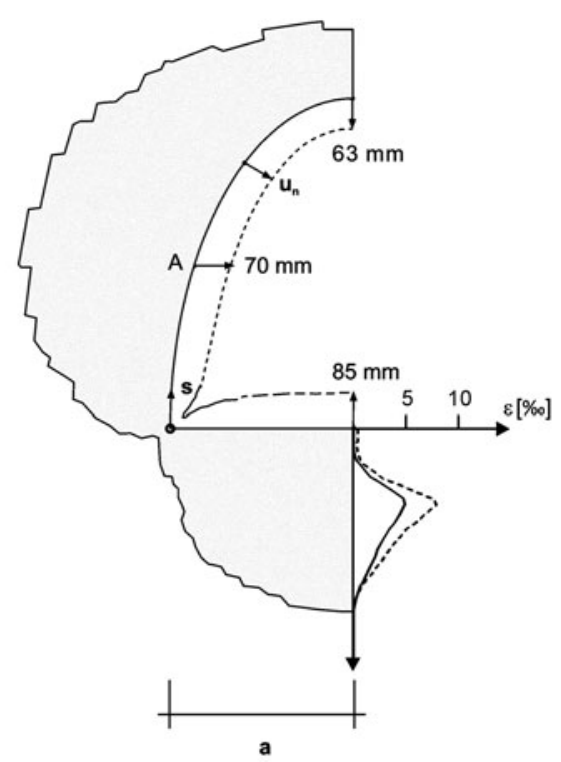

(c)

Fig. 7 Deep tunnel in hydrostatic initial stress field: considered tunnel cross sections of equal area, deformed cross section, extent of the plastic zone and plastic strain distribution $\left(\sigma_{s} * / \sigma_{0} *=0.2\right)$ 
The quantification of the functional relationship (14) is based upon a parametric study on the computational model of Fig. 1, $l_{x}=l_{y l}=l_{y u}=8 D, \quad \sigma_{\mathrm{V} 0}=\sigma_{\mathrm{H} 0}=\sigma_{0} \quad$ and $\sigma_{y u}=\sigma_{0}$. The numerical calculations were carried out for $v=0.25$ and for friction angles $\varphi$ of $20^{\circ}, 30^{\circ}$ and $40^{\circ}$. Figure $8 \mathrm{a}, \mathrm{b}$ shows the generalized convergence and the normalized difference between vertical and horizontal convergence, respectively, as functions of the ratio $\sigma_{s}^{*} / \sigma_{0}^{*}$ for the tunnel shapes and friction angles under consideration. The vertical convergence $V$ is equal to the change in the height of the cross section, while $H$ denotes the change of tunnel width half way up the cross section (Fig. 7, point A).

According to Fig. 8a, the differences in the response curves of the shapes investigated are rather small. The analytical solution underestimates the convergence particularly in the case of the horseshoe-shaped cross section. This is due to the statically favorable shape of a circular cross section, for which the GRC is valid in a strict mathematical sense.
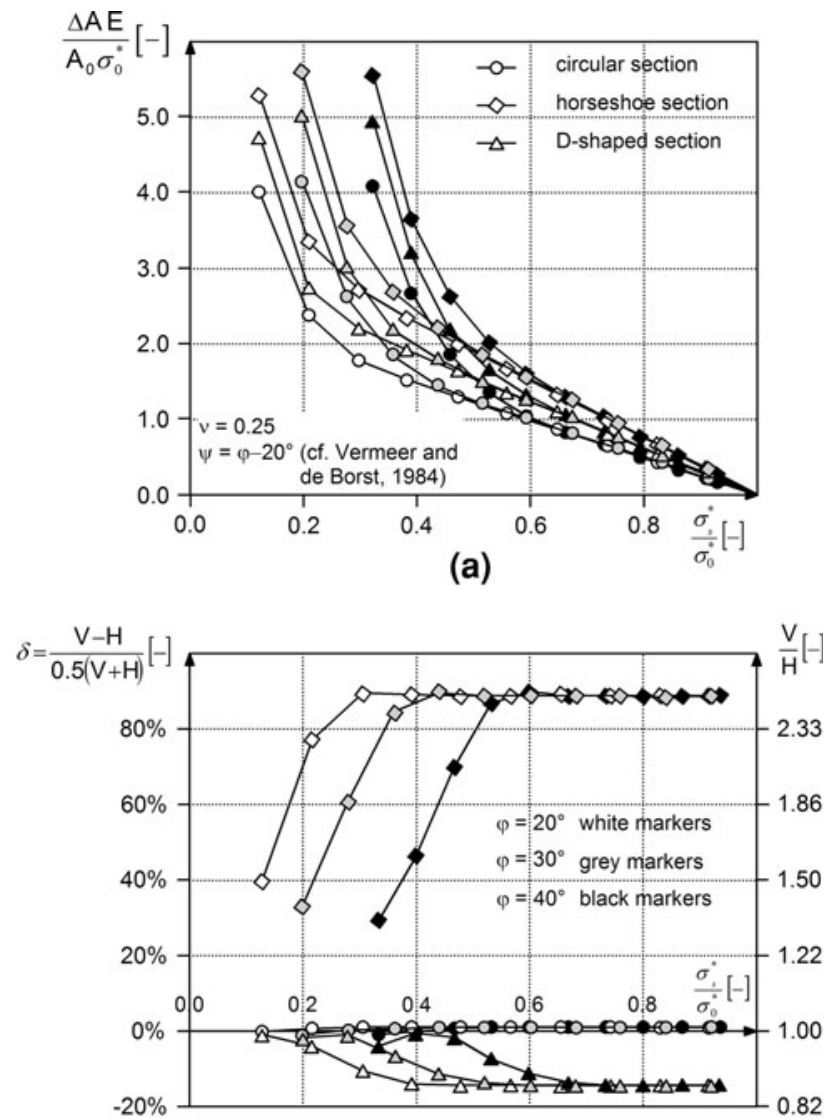

(b)

Fig. 8 Deep tunnel in hydrostatic initial stress field: a normalized average convergence, and $\mathbf{b}$ normalized difference between vertical and horizontal convergence as a function of the dimensionless relief coefficient $\sigma_{s} * / \sigma_{0} *$ and of the friction angle $\varphi$ for the tunnel cross sections of Fig. 7
Figure $8 \mathrm{~b}$ shows that at high normalized support pressures $\left(\sigma_{s}^{*} / \sigma_{0}^{*}>0.5\right)$, the difference $\delta$ between the horizontal and vertical convergence is constant and equal to its maximum value $(\delta=90 \%$ for the horseshoe section and $13 \%$ for the D-shaped section). At such higher support pressures, however, the response is linear and the deformations are small and not so important from the standpoint of practical design (within the elastic range, cf. Fig. 8a).

With decreasing support pressure $\sigma_{s}^{*} / \sigma_{0}^{*}$ the difference between the horizontal and vertical convergence also decreases (up to zero for the D-shaped section and up to $40 \%$ for the horseshoe-shaped section at $\left.\sigma_{s}^{*} / \sigma_{0}^{*}=0.2\right)$. The "homogenization" of the deformations at low support pressures is due to the extended plastification of the ground around the opening. As seen in Fig. 7, the shape of the plastic zone is independent from the tunnel shape at low support pressures.

\section{An Example with Several Deviations from Rotational Symmetry}

The aim of this example is to show that the GRC makes a reasonable job of approximating the numerically computed relationship between boundary displacements and support pressure, even if all of the assumptions underlying rotational symmetry are violated simultaneously. The example

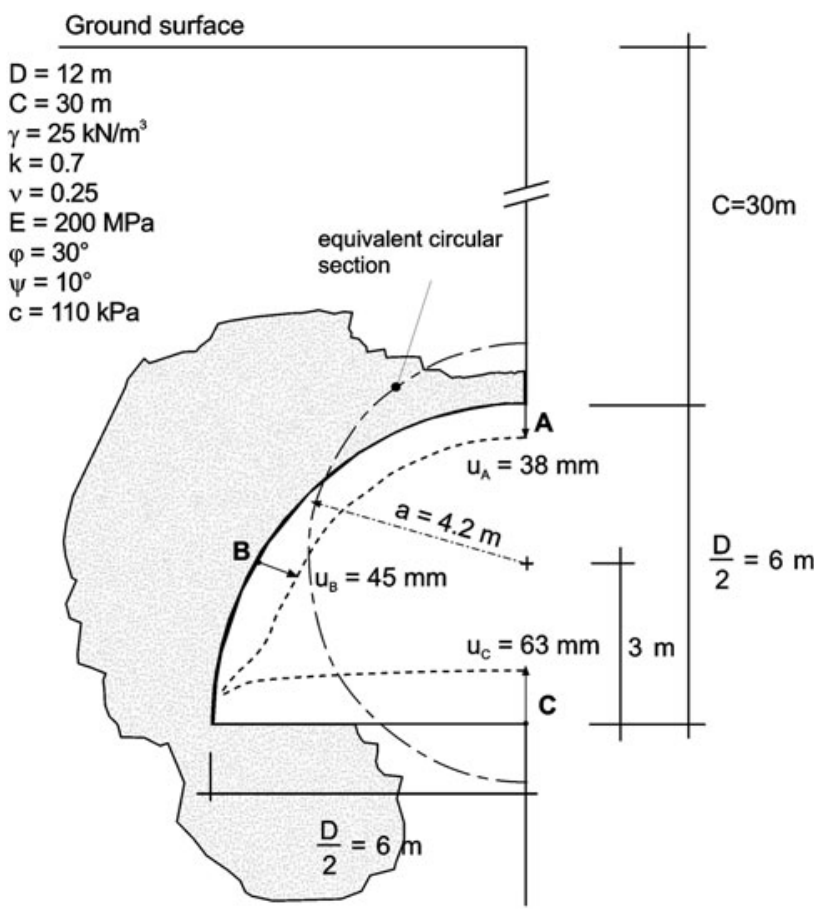

Fig. 9 Shallow unsupported non-cylindrical tunnel in non-hydrostatic initial stress field: considered cross section, equivalent circular cross section, extent of the plastic zone and deformed profile 
considers the top heading of a shallow tunnel $(D=12 \mathrm{~m}$, $C=30 \mathrm{~m}$, Fig. 9) in a non-hydrostatic initial stress field (with a lateral pressure coefficient of $k=0.7$ ). The FEM calculation was carried out using the computational model of Sect. 2. To obtain the relationship between displacement and support pressure, the stresses prevailing at the excavation boundary were reduced proportionally to their initial value. The GRC was calculated on the basis of an "equivalent" circular cross section (indicated by a dashed line in Fig. 9).

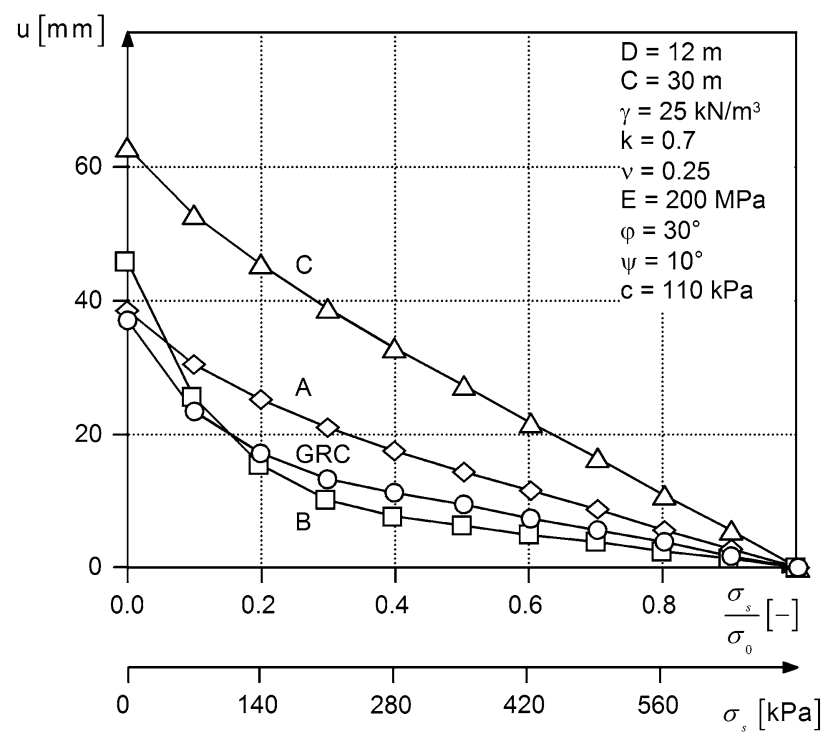

Fig. 10 Shallow non-cylindrical tunnel in non-hydrostatic initial stress field: GRC and radial displacement of points $\mathrm{A}, \mathrm{B}$ and $\mathrm{C}$ (Fig. 9) as a function of the normalized support pressure $\sigma_{s} / \sigma_{0}$ and of the support pressure $\sigma_{s}$

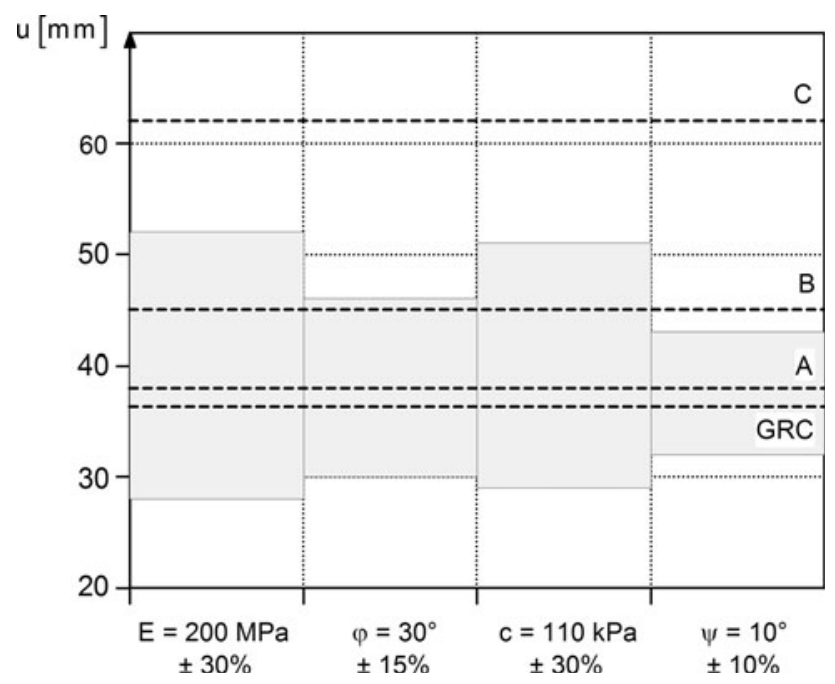

Fig. 11 Shallow unsupported non-cylindrical tunnel in non-hydrostatic initial stress field: Sensitivity of the closed-form solution (GRC) for the radial displacement with respect to the material constants $E, \varphi$, $c$ and $\psi$ and comparison with the radial displacement of the points A, $\mathrm{B}$ and $\mathrm{C}$ (Fig. 9)
Figure 9 shows the extent of the plastic zone and the deformed tunnel shape after complete unloading (an unsupported tunnel). Figure 10 shows the analytically computed GRC as well as the numerically calculated radial displacements of three characteristic points (denoted by $u_{\mathrm{A}}$, $u_{\mathrm{B}}$ and $u_{\mathrm{C}}$ in Fig. 9) as a function of the normalized support pressure $\sigma_{s} / \sigma_{0}$. Specifically for the GRC, which assumes uniform support, the absolute value of the support pressure $\sigma_{s}$ is also given. According to Fig. 10, the differences between the analytical solution and the numerical values are very small for the tunnel crown and the wall (points A and B). The closed-form solution produces a significant underestimate only of the heave of the flat floor (point C). Figure 11 shows, for the special case of an unsupported opening, the difference between analytical (GRC) and numerical displacement (A, B and C) in relation to the effect of a variation of the ground parameters according to Kovári (1986). The error introduced by the simplifying assumptions of the GRC is acceptable bearing in mind the sensitivity of the predictions with respect to the material constants.

\section{Conclusions}

The GRC approximates well to average tunnel convergence, even when the underlying assumption of rotational symmetry is violated to a considerable extent. The deviation between the analytical and numerical results is small compared to the uncertainties related to the assumptions concerning initial stress field and ground parameters (Fig. 11).

According to Sect. 2, overburdens of more than $40 \mathrm{~m}$ may be considered large in the sense that the effects of the non-uniformity of the initial stress field and the existence of the free surface can be neglected. Section 3 confirms earlier results from the literature, according to which the deviation between the analytical and numerical results is small for the usual values of the lateral pressure coefficient ( $k=0.7-1.5)$ and for uniaxial compressive strengths $f_{\mathrm{c}}<\sigma_{0}$. This is true even in the case of non-circular tunnel cross sections and overstressed ground $\left(\sigma_{s}^{*} / \sigma_{0}^{*}<0.2\right.$, Sect. 4) or even if all assumptions underlying the GRC are violated simultaneously within certain limits (Sect. 5).

It should be emphasized that these conclusions as to the predictive quality of the rotational symmetric model are true only with respect to average convergence and do not apply to the loading and stresses developing in a lining. Deviations from rotational symmetry lead to qualitatively different stressing of the lining because bending moments and shear forces develop in addition to the axial forces. In such cases, particularly if the tunnel has a non-circular cross section or a non-uniform support, numerical calculations are indispensable for assessing the lining. 


\section{References}

Anagnostou G (2001) Two shallow tunnels in soft ground. In: Proceedings of AITES-ITA 2001 WTC, Milano, 21-28 June 2001

Anagnostou G, Kovári K (1993) Significant parameters in elastoplastic analysis of underground openings. J Geotech Eng 119(3): 401-419

Bernaud D, Rousset G (1996) The 'new implicit method' for tunnel analysis. Int J Numer Anal Methods Geomech 20:673-690

Caquot A (1934) Equilibre des massifs a frottement interne. GauthierVillars, Paris

Carranza-Torres C, Fairhurst C (2000) Application of the convergence-confinement method of tunnel design to rock masses that satisfy the Hoek-Brown failure criterion. Tunn Undergr Space Technol 15(2):187-213

Curran JH, Hammah RE, Yacoub TE (2003) A two-dimensional approach for designing tunnel support in weak rock. In: Proceedings of the 56th Canadian Geotechnical Conference, Winnepeg

Detournay E (1983) Two-dimensional elastoplastic analysis of a deep cylindrical tunnel under non-hydrostatic loading, $\mathrm{PhD}$ dissertation, University of Minnesota

Detournay E, Fairhurst C (1987) Two-dimensional elastoplastic analysis of a long, cylindrical cavity under non-hydrostatic loading. Int J Rock Mech Min Sci 24(4):197-211

Eisenstein Z, Branco P (1991) Convergence-confinement method in shallow tunnels. Tunn Undergr Space Technol 6(3):343-346
Gonzáles-Nicieza C, Álvarez-Vigil AE, Méndez-Díaz A, GonzálesPalacio C (2008) Influence of the depth and shape of a tunnel in the application of the convergence-confinement method. Tunn Undergr Space Technol 23:25-37

Graziani A, Boldini D, Ribacchi R (2005) Practical estimate of deformations and stress relief factors for deep tunnels supported by shotcrete. Rock Mech Rock Eng 38(5):345-372

Kovári K (1986) Rock deformation problems when using full-facing cutting equipment in rock, part 2. Tunnel 4/86, 289-298, Bertelsmann Fachzeitschriften GmbH Gütersloh

Oreste P (2009) The convergence-confinement method: roles and limits in modern geomechanical tunnel design. Am J Appl Sci 6(5):757-771

Panet M, Guellec P (1974) Contribution à l'étude du soutènement derrière le front de taille. In: Proceedings of the 3rd Congr ISRM, Denver, 2, part B, pp 1130-1134

Poulos HG, Davis EH (1974) Elastic solutions for soil and rock mechanics. John Wiley and Sons, New York

Svoboda T, Mašín D (2010) Convergence-confinement method for simulating NATM tunnels evaluated by comparison with full 3D simulations. In: Proceedings of the 11th International Conference on Underground Construction, Prague, pp 795-801

Vermeer PA, de Borst R (1984) Non-associated plasticity for soils, concrete and rock. Heron 29(3):1-64

Vermeer A, Ruse N, Marcher T (2002) Tunnel heading stability in drained ground. Felsbau 20(6):8-18 